\title{
Nononcologic Applications of PET/CT and PET/MRI in Musculoskeletal, Orthopedic, and Rheumatologic Imaging: General Considerations, Techniques, and Radiopharmaceuticals
}

\author{
Ali Gholamrezanezhad ${ }^{1,2}$, Kyle Basques $^{3}$, Ali Batouli ${ }^{4}$, Mojtaba Olyaie $^{4}$, George Matcuk ${ }^{1}$, Abass Alavi ${ }^{5}$, \\ and Hossein Jadvar ${ }^{6}$ \\ ${ }^{1}$ Division of Musculoskeletal Imaging, Department of Radiology, Keck School of Medicine, University of Southern California, Los \\ Angeles, California; ${ }^{3}$ Division of Musculoskeletal Imaging, Department of Radiology, University Hospitals of Cleveland, Case \\ Western Reserve University, Cleveland, Ohio; ${ }^{4}$ Department of Radiology, Allegheny General Hospital, Pittsburgh, Pennsylvania; \\ ${ }^{5}$ Division of Nuclear Medicine, Department of Radiology, Hospital of the University of Pennsylvania, Philadelphia, Pennsylvania; \\ and ${ }^{6}$ Division of Nuclear Medicine, Department of Radiology, Keck School of Medicine, University of Southern California, Los \\ Angeles, California
}

\begin{abstract}
PET is often underutilized in the field of musculoskeletal imaging, with key reasons including the excellent performance of conventional musculoskeletal MRI, the limited spatial resolution of PET, and the lack of reimbursement for PET for nononcologic musculoskeletal indications. However, with improvements in PET/CT and PET/MRI over the last decade as well as an increased understanding of the pathophysiology of musculoskeletal diseases, there is an emerging potential for PET as a primary or complementary modality in the management of rheumatologic and orthopedic patients. Specific advantages of PET include the convenience of whole-body imaging in a single session, the relative resilience of the modality compared with CT and MRI in the imaging of metallic implants, the ability to evaluate deep joints not amenable to palpation, and the potential for improved specificity of diagnosis with novel radiopharmaceuticals. In this review, we discuss multiple radiopharmaceuticals and technical considerations for PET/CT and PET/MRI that can be used in imaging of nontumoral bone and soft-tissue disorders. Both PET/CT and PET/MRI hold significant promise in the field of musculoskeletal imaging, and with further radiopharmaceutical development and clinical research, these hybrid modalities can potentially transform the current management of patients with orthopedic and rheumatologic disease.
\end{abstract}

Key Words: PET/CT; PET/MRI; rheumatoid arthritis; septic arthritis; osteomyelitis; polymyalgia rheumatica

J Nucl Med Technol 2018; 46:33-38

DOI: $10.2967 /$ jnmt.117.198663

\footnotetext{
Received Jul. 8, 2017; revision accepted Sep. 22, 2017.

For correspondence or reprints contact: Ali Gholamrezanezhad, Division of Musculoskeletal Imaging, Department of Radiology, Keck School of Medicine, University of Southern California, 1520 San Pablo St., Los Angeles, CA 90033.

E-mail: a.gholamrezanezhad@yahoo.com

Published online Nov. 10, 2017.

COPYRIGHT (c) 2018 by the Society of Nuclear Medicine and Molecular Imaging.
}

$\mathbf{P}$ ET was introduced 4 decades ago and has rapidly become the standard of care for the diagnosis and monitoring of a wide range of diseases, particularly in the field of oncology. In addition, as a metabolic and molecular imaging modality, PET has significantly advanced our understanding of normal human physiology as well as pathophysiology $(1,2)$. PET is now most commonly used as a hybrid modality, typically combined with CT or MRI for better anatomic localization and attenuation correction purposes (3-5).

Despite the widespread application of PET/CT and PET/ MR for oncology, the role of PET imaging in musculoskeletal disorders, especially nononcologic applications, has not been widely appreciated. PET has traditionally been underutilized in musculoskeletal imaging for several reasons, such as the limited spatial resolution of PET and the excellent performance of musculoskeletal MRI, a modality that does not use ionizing radiation. However, with improvements in PET/CT and PET/MRI over the last decade as well as increased understanding of the pathophysiology of musculoskeletal diseases, there is an emerging potential for PET as a primary or complementary modality in the management of rheumatologic and orthopedic patients. In fact, the low metabolic activity of osseous and tendinous structures is an advantage for detecting pathology in the skeletal system, because of the low physiologic background activity leading to a high target-to-background ratio in imaging of these disease processes. In this review, we discuss the technical details of, and different radiopharmaceuticals for, PET/CT and PET/MRI in the management of nonneoplastic musculoskeletal diseases.

\section{PET RADIOPHARMACEUTICALS}

PET is a powerful molecular imaging technique in which a positron-emitting radiotracer is administered to a patient and then subsequent imaging visualizes the in vivo distribution 
and kinetics of that radiotracer. Multiple PET radiopharmaceuticals have been developed that interact with biologic systems, such as radiotracers studying glucose, amino acid, and nucleotide metabolism; cellular receptors and transporters; and bone and cell turnover metabolites.

${ }^{18} \mathrm{~F}-\mathrm{FDG}$ is by far the most common radiotracer used in clinical PET imaging. Its biodistribution follows the pattern of in vivo glucose metabolism, with increased glucose metabolism typically associated with neoplastic and inflammatory pathologies. Increased ${ }^{18} \mathrm{~F}$-FDG uptake is indeed seen both in neoplastic cells and in cellular elements of inflammation, such as macrophages, fibroblasts, neutrophils, and capillaries $(6,7) .{ }^{18} \mathrm{~F}-\mathrm{FDG}$ continues to be widely used because of its availability and low cost, as well as the wealth of evidence and years of experience demonstrating its clinical utility. However, ${ }^{18}$ F-FDG imaging suffers from the disadvantage of relatively limited specificity. Although this is a major limitation in the imaging evaluation of neoplastic disorders, it probably is not a major issue in the imaging approach to nonneoplastic disorders of the musculoskeletal system, where the diagnosis of diseases relies on mainly nonimaging clinical and serologic findings. In fact, in these cases, the high sensitivity of ${ }^{18}$ F-FDG can be an advantage in identifying mild and clinically silent stages of the disease.

${ }^{18} \mathrm{~F}-\mathrm{NaF}$ is a Food and Drug Administration-approved positron-emitting radiotracer used as a marker of osteoblastic activity. The main advantage of this radiotracer is that its uptake is minimal in bone marrow and its activity originates almost exclusively in the cortical bone. Uptake of ${ }^{18} \mathrm{~F}-\mathrm{NaF}$ in the skeletal system is flow-dependent and consists of fast chemisorption on hydroxyapatite crystals, forming fluoroapatite. ${ }^{18} \mathrm{~F}-\mathrm{NaF}$ PET is a highly sensitive imaging probe for the detection of osseous metastases and other nonneoplastic bone lesions, such as traumatic (Fig. 1) and metabolic pathologies (8). Uptake of ${ }^{18} \mathrm{~F}-\mathrm{NaF}$ is up to 10 times higher in osteoblastic lesions than in normal bone, leading to excellent image contrast. Contrast is further improved by the fact that ${ }^{18} \mathrm{~F}-\mathrm{NaF}$ does not bind to plasma protein and thus has fast renal excretion and therefore low background activity. It has been shown that ${ }^{18} \mathrm{~F}-\mathrm{NaF}$ PET/CT offers a higher sensitivity than MRI in the detection of osteoblastic bone lesions $(9,10)$. Combining ${ }^{18} \mathrm{~F}-\mathrm{NaF}$ PET with MRI is a powerful approach that incorporates the high sensitivity of PET and the robust specificity of MRI for early diagnosis and accurate posttreatment follow-up of osteoblastic lesions (11).

Several other PET radiotracers are being studied for use in the musculoskeletal system. There has been increased interest in developing radiotracers with increased specificity compared with ${ }^{18} \mathrm{~F}-\mathrm{FDG}$ and ${ }^{18} \mathrm{~F}-\mathrm{NaF}$, as well as radiotracers using shorter-lived positron-emitting radioisotopes that take advantage of newer PET equipment to limit radiation exposure and reduce image acquisition time (12).

\section{${ }^{68} \mathrm{Ga}-$ Citrate and ${ }^{68} \mathrm{Ga}-$ Transferrin}

${ }^{68} \mathrm{Ga}$-citrate and ${ }^{68} \mathrm{Ga}$-transferrin were originally used for tumor imaging but have recently been studied for possible

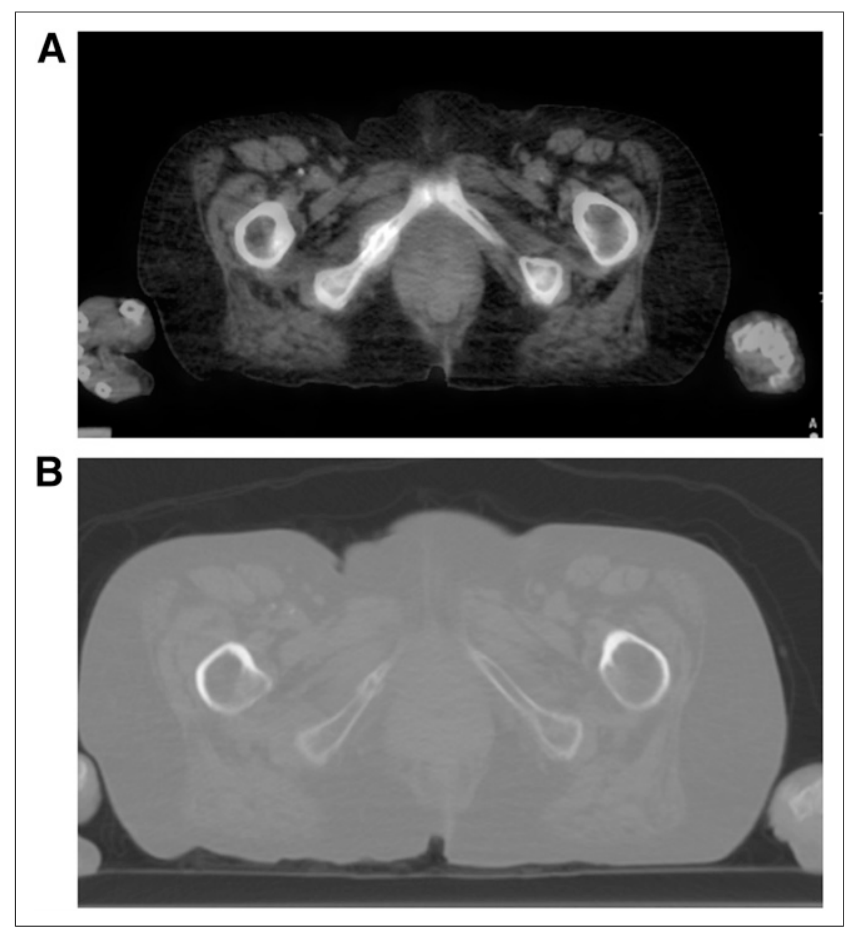

FIGURE 1. 67-y-old woman with bilateral breast cancer underwent ${ }^{18} \mathrm{~F}-\mathrm{NaF}$ PET/CT for evaluation of osseous metastasis. Focus of intensely increased radiotracer uptake in right ischiopubic junction (A) corresponded to healing fracture identified on lowdose CT (B).

imaging of inflammation and infection in the musculoskeletal system (13). ${ }^{68} \mathrm{Ga}$ has a half-life of $68 \mathrm{~min}$, with high blood-pool activity and liver and bone uptake but low softtissue activity. It binds to lactoferrin, which is present in high concentrations in neutrophils and abscess fluid as well as in siderophores produced by different infectious microorganisms $(12,14)$. In clinical studies, these agents have been successful in detecting the infection site as early as $30 \mathrm{~min}$ after injection, but current protocols allow 60 min after injection to reduce background activity and improve image quality. High background activity in the thorax and upper abdomen may interfere with detecting thoracic and upper abdominal lesions; therefore, ${ }^{68} \mathrm{Ga}$-citrate and ${ }^{68} \mathrm{Ga}$-transferrin are most useful for lower abdominal and extremity infection sites. Imaging with these ${ }^{68} \mathrm{Ga}$ agents is useful not only for the initial diagnosis of infection but also for planning surgery, monitoring treatment (Fig. 2), and differentiating prosthetic infection from aseptic loosening of a prosthesis (15).

\section{${ }^{68}$ Ga-DOTA-Sialic Acid-Binding Immunoglobulin-like Lectin-9 ( ${ }^{68} \mathrm{Ga}$-Siglec-9)}

${ }^{68} \mathrm{Ga}-\mathrm{Siglec}-9$ is a PET radiotracer introduced for the assessment of synovitis and the in vivo imaging of inflammation. Siglec-9 is a leukocyte ligand of vascular adhesion protein 1, which, during inflammatory processes, rapidly translocates from the intracellular space to the endothelial surface of cells, including vessels in human rheumatoid synovium (16). This is an important step in the regulation 


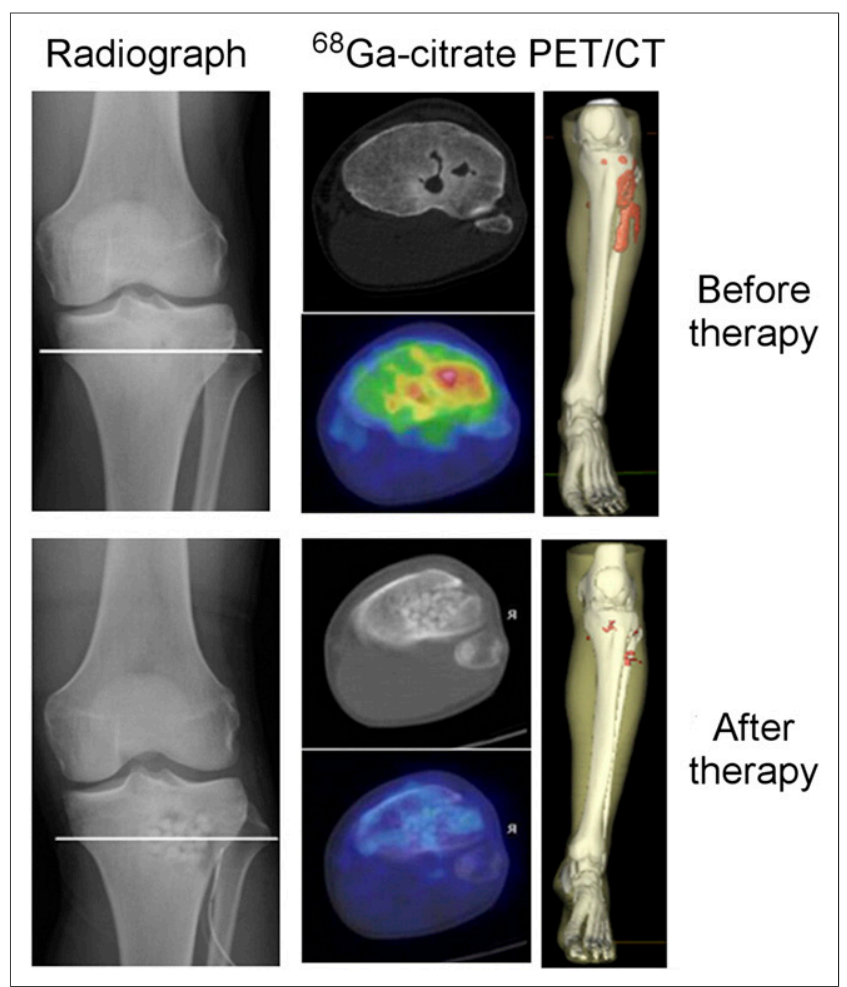

FIGURE 2. Comparison of pre- and posttreatment ${ }^{68} \mathrm{Ga}$-citrate PET/CT scans in patient with acute osteomyelitis. Pretreatment scan showed increased radiotracer uptake involving proximal tibia. Posttreatment scan showed no significant uptake, representing complete response to treatment. (Reprinted from (13).)

of leukocyte migration to sites of inflammation (17). However, as a PET radiotracer, ${ }^{68} \mathrm{Ga}$-Siglec-9 is somewhat nonspecific and detects vascular adhesion protein 1 not only in the vasculature at sites of inflammation but also in cancer tissues (18). ${ }^{18} \mathrm{~F}$-labeling of Siglec-9 peptide has also been performed in the preclinical setting $(19,20)$.

\section{${ }^{89} \mathrm{Zr}$-Labeled Rituximab}

${ }^{89} \mathrm{Zr}$-labeled rituximab has been introduced as a specific radiotracer for noninvasive B-cell imaging by PET/CT (17). The CD20+ B-cell count in the lymph nodes of subjects who underwent ${ }^{89} \mathrm{Zr}$-rituximab PET imaging correlated positively with quantitative lymph node PET data. B cells are key players in the pathogenesis of autoimmune inflammatory arthropathies, such as rheumatoid arthritis and systemic lupus erythematosus. ${ }^{89} \mathrm{Zr}$-rituximab has been successfully used in identifying potential responders before treatment (Fig. 3) (17).

\section{${ }^{11} \mathrm{C}$-Methionine}

${ }^{11} \mathrm{C}$-methionine is used for PET imaging of amino acid metabolism. It was originally used to try to distinguish neoplastic from inflammatory lesions $(12,21)$. However, it was subsequently found to accumulate in both categories of lesions. Currently, the radiotracer is being studied for the imaging of musculoskeletal infectious and inflammatory processes (22). Soft-tissue and bone uptake of ${ }^{11} \mathrm{C}$-methionine is relatively low in the extremities, making it a promising radiotracer for evaluation of limb musculoskeletal infections, particularly in the pediatric and adolescent population $(12,23)$.

\section{${ }^{11}$ C-PK11195}

${ }^{11} \mathrm{C}-\mathrm{PK} 11195$ is a radiolabeled form of PK11195, a specific ligand for the peripheral benzodiazepine receptor, which is highly expressed on activated mononuclear phagocytic cells $(12,24)$. This radiotracer has primarily been investigated for the diagnosis of neuroinflammation (25), but more recent work has proposed a role in nonneurologic inflammation, such as inflammation of vascular structures and periprosthetic soft tissues in animal models $(24,26)$.

\section{1-(2'-Deoxy-2'-Fluoro- $\beta$-D-Arabinofuranosyl)- 5-lodouracil (FIAU)}

FIAU has been found to be a substrate for bacterial thymidine kinase. ${ }^{124}$ I-FIAU has been tested in humans as an infection-imaging radiotracer $(27,28)$.

\section{${ }^{18} \mathrm{~F}$-Labeled Leukocytes}

${ }^{18} \mathrm{~F}$-labeled leukocytes have been shown to be effective in the diagnosis of various infectious and inflammatory pathologies. The underlying mechanism of labeled leukocyte imaging is based on chemotaxis exerted on activated leukocytes by chemoattractants, resulting in cell-bound radionuclide trafficking from the blood-pool compartment to the lesion. The use of ${ }^{18} \mathrm{~F}$ labeling is facilitated by the avidity of inflammatory cells for ${ }^{18}$ F-FDG $(29,30)$.

\section{Integrin $\alpha_{v} \beta_{3}$}

Integrin $\alpha_{v} \beta_{3}$ has been suggested as a target for imaging of angiogenesis, as it is expressed on activated endothelial cells. ${ }^{18} \mathrm{~F}$-galacto-RGD (arginine-glycine-aspartate) has been introduced as a radiotracer for PET imaging of integrin $\alpha_{v} \beta_{3}$ expression. Although this tracer is mainly studied for oncologic applications, some authors have suggested that it can be also used for the evaluation of inflammatory disorders (31).

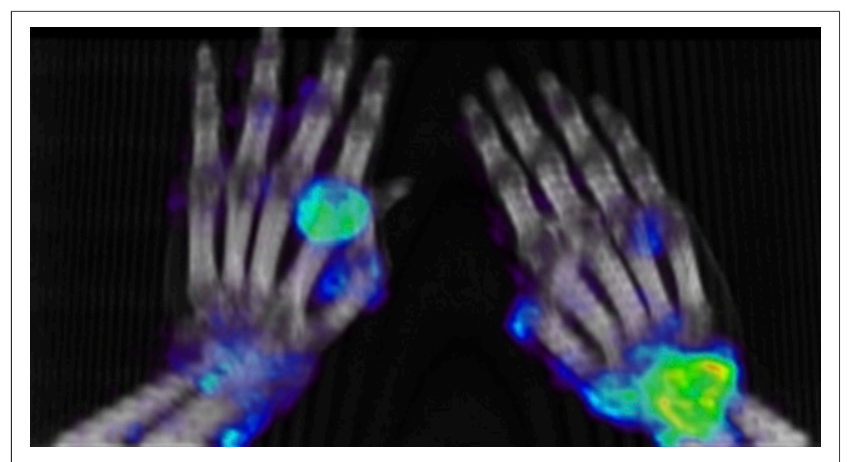

FIGURE 3. ${ }^{89} \mathrm{Zr}$-rituximab PET/CT scan of patient with active rheumatoid arthritis, demonstrating increased radiotracer uptake involving left second metacarpophalangeal joint and right wrist. (Image courtesy of Dr. Conny van der Laken, Afdeling Reumatologie, VU Universitair Medisch Centrum, Amsterdam.) 


\section{Others}

Several other radiotracers, such as ${ }^{11} \mathrm{C}$-choline, ${ }^{11} \mathrm{C}$-tyrosine, and ${ }^{18} \mathrm{~F}$-fluorothymidine, have also been studied for PET imaging of the musculoskeletal system (31). However, their main applications were the study of neoplastic disorders, which is beyond the scope of this review.

\section{IMAGING PROTOCOL}

A detailed discussion of PET/CT and PET/MRI protocols is beyond the scope of this article. Generally, when ${ }^{18} \mathrm{~F}-$ FDG PET is performed, imaging of the patient $60 \mathrm{~min}$ after radiotracer injection is recommended. However, several modifications in imaging protocols have been used to increase the specificity of ${ }^{18} \mathrm{~F}-\mathrm{FDG}$ PET for nononcologic indications.

Dual-time-point PET imaging has been suggested for the diagnosis of osteomyelitis. It has been shown that dualtime-point imaging with ${ }^{18} \mathrm{~F}-\mathrm{FDG}$ is more accurate than single-time-point imaging in the differentiation of benign lesions from malignant neoplastic pathologies $(32,33)$. Sahlmann et al. demonstrated that dynamic dual-time-point ${ }^{18}$ F-FDG PET elicits a characteristic pattern in chronic osteomyelitis-like that in inflammatory processes in other locations-that differentiates it from neoplastic pathologies. In chronic osteomyelitis, the $\mathrm{SUV}_{\max }$ of scans obtained between 30 and 90 min after injection remains stable or decreases, with a median decrease of $6 \%$, whereas in malignant lesions, the $\mathrm{SUV}_{\text {max }}$ and $\mathrm{SUV}_{\text {mean }}$ between 30 and 90 min after injection both increase (32).

Brown et al. also tested this hypothesis on an experimental rabbit model of postsurgical osteomyelitis to distinguish between acute infection and sterile postsurgical inflammation (34). In their study, images were acquired 7 and $14 \mathrm{~d}$ after surgical intervention, with continuous PET image acquisition for $90 \mathrm{~min}$ after ${ }^{18} \mathrm{~F}$-FDG administration. However, their findings were not promising, and they concluded that in the complicated clinical context of acute postsurgical and posttraumatic inflammation versus infection, the diagnostic accuracy of ${ }^{18} \mathrm{~F}$-FDG PET is limited. This limitation is due to the fact that increased glucose utilization is the main basis of PET imaging and that this type of hypermetabolism is generally characteristic of all inflammatory processes, including infectious pathologies, but is not specific to any of them. The conclusion of Brown et al. was concordant with what was previously concluded by Kälicke et al. from their clinical study on 21 patients with suspected osteomyelitis-that PET is not able to differentiate between postsurgical reactive changes and further infection in the early postoperative phase (35).

\section{QUANTITATIVE PET IMAGING}

Quantification of the metabolic data obtained from PET images offers several benefits in the diagnostic management of diseases, including assessment of response to therapy in serial and follow-up imaging. Several methods of quantifica- tion have been proposed, categorized into semiquantitative measures and absolute quantitative analysis. Semiquantitative methods include measures such as SUV and SUV corrected for factors such as lean body mass, target-to-background ratio, and other variants. They are routinely used in the daily clinical practice of nuclear medicine as simple but practical and objective indicators of the metabolic activity of the lesion. However, these semiquantitative measures are prone to interreader and intrareader variability (36).

Absolute quantitative techniques use various mathematic models on PET data, including nonlinear regression and Patlak-Gjedde graphical analysis, and may require dynamic or parametric whole-body PET image acquisition. In dynamic image acquisition, time series of PET data are dynamically acquired, enabling simulation of physiologic processes through tracer kinetic modeling. The models may use region-of-interest-based kinetic analysis or voxel-based kinetic modeling (36), the detail of which is beyond the scope of this review. More sophisticated techniques for the quantification of PET data have allowed for specialized applications such as static and dynamic ${ }^{18} \mathrm{~F}-\mathrm{NaF}$ PET quantification of tracer plasma clearance (inhibition constant) in osteoporosis. Compartmental and noncompartmental machine learning models have already been tested in animal models (37), and there is great potential for the use of quantitative PET to better understand biologic systems.

\section{PET/CT VERSUS PET/MRI}

The hybrid modality PET/CT has become widely used and widely available since its introduction, with combination of the two modalities providing major advantages such as improved anatomic localization and improved attenuation correction. PET/CT is currently the standard of care for most clinical applications of PET imaging.

Technologic advances during the past decade, including the development of digital detector technology that is not disturbed by the magnetic field, has allowed the development of PET/MRI. These advances also include Dixon sequences, which allow acquisition of whole-body MRI in a reasonable time frame. PET/MRI, as a hybrid modality, provides several advantages over PET/CT, particularly with respect to evaluation of the musculoskeletal system. For example, the lower ionizing radiation exposure of PET/ MRI than of PET/CT is particularly important for the pediatric population and for young adults who may need repeated follow-up imaging studies for an extended period. In these cases, PET/MRI can spare patients from a significant amount of radiation (1).

More importantly, PET/MRI provides higher soft-tissue contrast than PET/CT, which is crucial for appropriate diagnosis of ligamentous, tendinous, and muscular pathologies $(1,2)$. In addition, because MRI with multisequence multiparametric protocoling is usually required for the diagnostic work-up of these soft-tissue pathologies, PET/MRI 
can potentially serve as a 1-stop shop for patient work-up, where both diagnostic MRI of the pertinent anatomy and whole-body PET/MRI can be performed during the same imaging session.

In some applications, PET/MRI can be superior to PET/ CT by way of its more accurate coregistration and better MRI-based motion correction of PET data (1). Although no head-to-head comparisons of PET/CT and PET/MRI have been reported for musculoskeletal applications, the currently available PET/MRI machines generally provide image quality comparable to PET/CT (3-5). In addition, no significant negative effects of the PET hardware on MR image quality and machine functionality have been identified.

However, compared with PET/CT, PET/MRI does have some disadvantages in the evaluation of musculoskeletal pathologies. For example, attenuation correction continues to be better for PET/CT than for PET/MRI. PET/CT is also much more widely available and is lower in cost. PET/MRI scanners not only are more expensive but generally have a lengthier image acquisition time that further increases the cost. MRI of the extremities also often needs special coils, such as those for the knees or wrists. These coils are often an additional expense, and third-party coils are not necessarily PET-friendly. Also, some patients have contraindications to MRI, including permanent pacemakers, intraaortic balloon pumps, and left and right ventricular assist devices, but can be safely imaged by PET/CT.

There are also differences in the quantification of ${ }^{18} \mathrm{~F}$ FDG uptake and metabolic activity using the measure of SUV. Although SUV can be used in PET/MRI, the values are scanner-dependent and not entirely comparable to those obtained from PET/CT. MRI also is weaker than CT in the evaluation of cortical bone, though newly introduced techniques such as ultrashort echo time sequences may help to overcome this difference. However, such sequences also have their own drawbacks, including artifacts at larger fields of view and longer image acquisition times $(2,38,39)$. No experience with these sequences on PET/MRI has been published, and more investigation is warranted to confirm the usefulness in PET/MRI for musculoskeletal applications.

Both PET/CT techniques and PET/MRI techniques continue to advance. At present, the two are considered comparable for most clinical applications, though evidence is building that PET/MRI may be superior for certain applications, such as the confident diagnosis of osteomyelitis (40). PET/CT remains the more commonly performed examination because of its greater availability and lower cost (41).

\section{FUTURE PERSPECTIVES}

Multiple PET radiotracers with potential applications in the musculoskeletal system have been developed. Many of these radiotracers have been tested only in animal models, but there are many promising opportunities for future human clinical trials using agents that have favorable diagnostic performance and safety profiles.

The use of hybrid PET/MRI is rapidly evolving in the diagnostic management of different disorders, with the major advantage being combination of the morphologic information of MRI and the functional information of PET $(40,42)$. PET/MRI in the management of musculoskeletal disorders is currently a hot topic for investigation, with scarce reports of its use in nononcologic applications. The combination of PET using ${ }^{18} \mathrm{~F}-\mathrm{FDG}$ or other more specialized radiotracers with novel MRI sequences tailored for the musculoskeletal system raises numerous exciting possibilities.

\section{CONCLUSION}

As investigations of the use of PET in the musculoskeletal system increase, measurement of the diagnostic performance and clinical outcomes of PET modalities will continue to be important, particularly in children, for whom exposure to ionizing radiation will always remain a drawback. Comparisons of PET with competing modalities that use less or no ionizing radiation must be performed to ensure appropriate care.

\section{DISCLOSURE}

No potential conflict of interest relevant to this article was reported.

\section{REFERENCES}

1. Andersen KF, Jensen KE, Loft A. PET/MR imaging in musculoskeletal disorders. PET Clin. 2016;11:453-463.

2. Bashir U, Mallia A, Stirling J, et al. PET/MR in oncological imaging: state of the art. Diagnostics (Basel). 2015;5:333-357.

3. Giraudo C, Raderer M, Karanikas G, et al. ${ }^{18} \mathrm{~F}$-Fluorodeoxyglucose positron emission tomography/magnetic resonance in lymphoma: comparison with ${ }^{18} \mathrm{~F}$ fluorodeoxyglucose positron emission tomography/computed tomography and with the addition of magnetic resonance diffusion-weighted imaging. Invest Radiol. 2016;51:163-169.

4. Platzek I, Beuthien-Baumann B, Langner J, et al. PET/MR for therapy response evaluation in malignant lymphoma: initial experience. MAGMA. 2013;26:49-55.

5. Platzek I. ${ }^{18} \mathrm{~F}$-fluorodeoxyglucose PET/MR imaging in lymphoma. PET Clin. 2016;11:363-373.

6. Kubota K, Ito K, Morooka M, et al. FDG PET for rheumatoid arthritis: basic considerations and whole-body PET/CT. Ann N Y Acad Sci. 2011;1228:29-38.

7. Matsui T, Nakata N, Nagai S, et al. Inflammatory cytokines and hypoxia contribute to ${ }^{18} \mathrm{~F}$-FDG uptake by cells involved in pannus formation in rheumatoid arthritis. J Nucl Med. 2009;50:920-926.

8. Tarnawska-Pierścińska M, Hołody Ł, Braziewicz J, Królicki L. Bone metastases diagnosis possibilities in studies with the use of ${ }^{18} \mathrm{~F}-\mathrm{NaF}$ and ${ }^{18} \mathrm{~F}-\mathrm{FDG}$. Nucl Med Rev Cent East Eur. 2011;14:105-108.

9. Pampaloni MH, Nardo L. PET/MR radiotracer beyond ${ }^{18}$ F-FDG. PET Clin. 2014;9:345-349.

10. Mosavi F, Johansson S, Sandberg DT, et al. Whole-body diffusion-weighted MRI compared with ${ }^{18} \mathrm{~F}-\mathrm{NaF}$ PET/CT for detection of bone metastases in patients with high-risk prostate carcinoma. AJR. 2012;199:1114-1120.

11. Ouvrier MJ, Vignot S, Thariat J. State of the art in nuclear imaging for the diagnosis of bone metastases. Bull Cancer. 2013;100:1115-1124.

12. Nielsen OL, Afzelius P, Bender D, et al. Comparison of autologous ${ }^{111} \mathrm{In}-$ leukocytes, ${ }^{18} \mathrm{~F}$-FDG, ${ }^{11} \mathrm{C}$-methionine, ${ }^{11} \mathrm{C}$-PK11195 and ${ }^{68} \mathrm{Ga}$-citrate for diagnostic nuclear imaging in a juvenile porcine haematogenous staphylococcus aureus osteomyelitis model. Am J Nucl Med Mol Imaging. 2015;5: $169-182$. 
13. Nanni C, Errani C, Boriani L, et al. ${ }^{68} \mathrm{Ga}$-citrate PET/CT for evaluating patients with infections of the bone: preliminary results. J Nucl Med. 2010; 51:1932-1936.

14. Kumar V, Boddeti DK, Evans SG, Angelides S. ${ }^{68}$ Ga-citrate-PET for diagnostic imaging of infection in rats and for intra-abdominal infection in a patient. Curr Radiopharm. 2012;5:71-75.

15. Kumar V, Boddeti DK. ${ }^{68}$ Ga-radiopharmaceuticals for PET imaging of infection and inflammation. Recent Results Cancer Res. 2013;194:189-219.

16. Virtanen H, Autio A, Siitonen R, et al. ${ }^{68}$ Ga-DOTA-Siglec-9: a new imaging tool to detect synovitis. Arthritis Res Ther. 2015;17:308.

17. Bruijnen S, Tsang-A-Sjoe M, Raterman H, et al. B-cell imaging with zirconium89 labelled rituximab PET-CT at baseline is associated with therapeutic response 24 weeks after initiation of rituximab treatment in rheumatoid arthritis patients. Arthritis Res Ther. 2016;18:266.

18. Aalto K, Autio A, Kiss EA, et al. Siglec-9 is a novel leukocyte ligand for vascular adhesion protein-1 and can be used in PET imaging of inflammation and cancer. Blood. 2011;118:3725-3733.

19. Li XG, Autio A, Ahtinen H, et al. Translating the concept of peptide labeling with 5deoxy-5- $\left[{ }^{18} \mathrm{~F}\right]$ fluororibose into preclinical practice: ${ }^{18} \mathrm{~F}$-labeling of Siglec-9 peptide for PET imaging of inflammation. Chem Commun (Camb). 2013;49:3682-3684.

20. Li XG, Helariutta K, Roivainen A, Jalkanen S, Knuuti J, Airaksinen AJ. Using 5deoxy-5-[ $\left[{ }^{18} \mathrm{~F}\right]$ fluororibose to glycosylate peptides for positron emission tomography. Nat Protoc. 2014;9:138-145.

21. Zhao S, Kuge Y, Kohanawa M, et al. Usefulness of ${ }^{11} \mathrm{C}$-methionine for differentiating tumors from granulomas in experimental rat models: a comparison with ${ }^{18}$ F-FDG and ${ }^{18}$ F-FLT. J Nucl Med. 2008;49:135-141.

22. Roivainen A, Parkkola R, Yli-Kerttula T, et al. Use of positron emission tomography with methyl- ${ }^{11} \mathrm{C}$-choline and $2-{ }^{18} \mathrm{~F}$-fluoro-2-deoxy-D-glucose in comparison with magnetic resonance imaging for the assessment of inflammatory proliferation of synovium. Arthritis Rheum. 2003;48:3077-3084.

23. Harris SM, Davis JC, Snyder SE, et al. Evaluation of the biodistribution of ${ }^{11} \mathrm{C}$ methionine in children and young adults. J Nucl Med. 2013;54:1902-1908.

24. Pugliese F, Gaemperli O, Kinderlerer AR, et al. Imaging of vascular inflammation with [ $\left.{ }^{11} \mathrm{C}\right]-\mathrm{PK} 11195$ and positron emission tomography/computed tomography angiography. J Am Coll Cardiol. 2010;56:653-661.

25. Cagnin A, Gerhard A, Banati RB. In vivo imaging of neuroinflammation. Eur Neuropsychopharmacol. 2002;12:581-586.

26. Ren W, Muzik O, Jackson N, et al. Differentiation of septic and aseptic loosening by PET with both ${ }^{11} \mathrm{C}-\mathrm{PK} 11195$ and ${ }^{18} \mathrm{~F}-\mathrm{FDG}$ in rat models. Nucl Med Commun. 2012;33:747-756.
27. Bettegowda C, Foss CA, Cheong I, et al. Imaging bacterial infections with radiolabeled 1-(2'-deoxy-2'-fluoro-beta-D-arabinofuranosyl)-5-iodouracil. Proc Natl Acad Sci USA. 2005;102:1145-1150.

28. Diaz LA Jr, Foss CA, Thornton K, et al. Imaging of musculoskeletal bacterial infections by [ ${ }^{124}$ I]FIAU-PET/CT. PLoS One. 2007;2:e1007.

29. Dumarey N. Imaging with FDG labeled leukocytes: is it clinically useful? $Q J$ Nucl Med Mol Imaging. 2009;53:89-94.

30. Rini JN, Palestro CJ. Imaging of infection and inflammation with ${ }^{18}$ F-FDGlabeled leukocytes. Q J Nucl Med Mol Imaging. 2006;50:143-146.

31. Al-Ibraheem A, Buck AK, Beer AJ, Herrmann K. Alternative PET tracers in musculoskeletal disease. PET Clin. 2010;5:363-374.

32. Sahlmann CO, Siefker U, Lehmann K, Meller J. Dual time point 2-[ $\left.{ }^{18} \mathrm{~F}\right]$ fluoro2 -deoxyglucose positron emission tomography in chronic bacterial osteomyelitis. Nucl Med Commun. 2004;25:819-823.

33. Tian R, Su M, Tian Y, et al. Dual-time point PET/CT with F-18 FDG for the differentiation of malignant and benign bone lesions. Skeletal Radiol. 2009;38:451-458.

34. Brown TL, Spencer HJ, Beenken KE, et al. Evaluation of dynamic $\left[{ }^{18} \mathrm{~F}\right]-\mathrm{FDG}-$ PET imaging for the detection of acute post-surgical bone infection. PLoS One. 2012; 7:e41863.

35. Kälicke T, Schmitz A, Risse JH, et al. Fluorine-18 fluorodeoxyglucose PET in infectious bone diseases: results of histologically confirmed cases. Eur J Nucl Med. 2000;27:524-528.

36. Houshmand S, Salavati A, Hess S, Werner TJ, Alavi A, Zaidi H. An update on novel quantitative techniques in the context of evolving whole-body PET imaging. PET Clin. 2015;10:45-58.

37. Cheng C, Heiss C, Dimitrakopoulou-Strauss A, et al. Evaluation of bone remodeling with ${ }^{18} \mathrm{~F}$-fluoride and correlation with the glucose metabolism measured by ${ }^{18} \mathrm{~F}-\mathrm{FDG}$ in lumbar spine with time in an experimental nude rat model with osteoporosis using dynamic PET-CT. Am J Nucl Med Mol Imaging. 2013;3:118-128.

38. Chaudhry AA, Gul M, Gould E, Teng M, Baker K, Matthews R. Utility of positron emission tomography-magnetic resonance imaging in musculoskeletal imaging. World J Radiol. 2016;8:268-274.

39. Quick HH. Integrated PET/MR. J Magn Reson Imaging. 2014;39:243-258.

40. Signore A, Glaudemans AWJM, Gheysens O, Lauri C, Catalano OA. Nuclear medicine imaging in pediatric infection or chronic inflammatory diseases. Semin Nucl Med. 2017;47:286-303.

41. Gholamrezanezhad A, Chirindel A, Subramaniam R. Assessment of response to therapy. In: PET-CT and PET-MRI in Oncology: A Practical Guide. New York, NY: Springer; 2012:279-322.

42. Lee IS, Jin YH, Hong SH, Yang SO. Musculoskeletal applications of PET/MR. Semin Musculoskelet Radiol. 2014;18:203-216. 